\title{
Key Strategies for Overcoming Psychological Insulin Resistance in Adults with Type 2 Diabetes: The UK Subgroup in the EMOTION Study
}

\author{
Elemer G. Balogh • Magaly Perez-Nieves · Dachuang Cao • \\ Irene I. Hadjiyianni · Nadeem Ashraf • Urvi Desai · Frank J. Snoek • \\ Jackie A. Sturt
}

Received: March 11, 2020 / Published online: June 19, 2020

(C) The Author(s) 2020

\begin{abstract}
Introduction: Many patients with type 2 diabetes mellitus (T2DM) delay initiation of insulin therapy despite healthcare professional (HCP) advice. This phenomenon has been referred to as 'psychological insulin resistance' (PIR), and various contributing factors have been identified. Studies discussing approaches to overcoming PIR are lacking. Our aim was to identify the key strategies used by HCPs that most helped adults with T2DM and PIR in the UK to initiate insulin.
\end{abstract}

Digital Features To view digital features for this article go to https://doi.org/10.6084/m9.figshare.12423896.

E. G. Balogh · N. Ashraf

Eli Lilly and Company, Basingstoke, UK

M. Perez-Nieves $(\bowtie) \cdot$ D. Cao

Eli Lilly and Company, Indianapolis, IN, USA

e-mail: perez_magaly@lilly.com

I. I. Hadjiyianni

Lilly Deutschland GmbH, Bad Homburg, Germany

U. Desai

Analysis Group, Inc., Boston, MA, USA

F. J. Snoek

Amsterdam University Medical Centers, Vrije

Universiteit, Amsterdam, Netherlands

J. A. Sturt

Florence Nightingale Faculty of Nursing, Midwifery \& Palliative Care, King's College London, London, UK
Methods: As part of a global study, UK adults with T2DM and PIR were recruited $(N=125)$ to take a survey that included 38 HCP statements and actions about insulin initiation. Data assessed were perceived occurrence and helpfulness of these strategies in facilitating insulin initiation.

Results: The most helpful strategies involved demonstrating the injection process (e.g. HCP talked patient through the process of taking insulin [83.6\%]) and adopting a collaborative approach (HCP encouraged patient to contact the clinic immediately in case of any problems/ questions [80.5\%]). Additionally, HCPs highlighting the benefits of insulin (HCP explained that insulin was a natural substance needed by patient's body [81.2\%]) and allaying patients' concerns (HCP explained that patient might not have to take insulin forever [78.0\%]) helped patients initiate insulin. The least helpful action was HCPs repeatedly persuading patients to initiate insulin (40.9\%).

Conclusions: The study recommends key strategies that HCPs can adopt to help adults with T2DM overcome PIR in the UK.

\section{PLAIN LANGUAGE SUMMARY}

Many patients with type 2 diabetes (T2DM) are reluctant to start insulin therapy despite it being recommended by their doctor. This can 
lead to a delay in receiving effective treatment to control blood sugar. There are many reasons to explain this reluctance-which is also referred to as psychological insulin resistance (PIR)-including fear of injections and lack of understanding. EMOTION was a global study which set out to identify strategies to overcome PIR. It looked at 38 things, identified by people with diabetes, that doctors/nurses can do or say to encourage a patient to try insulin. Analysis of results for the 125 UK patients with T2DM who were reluctant to start insulin showed that the most helpful approach was demonstrating the injection procedure. Actually talking a patient through how to inject insulin and demonstrating how the pen works can help reduce their fears about the injection process. Adopting a collaborative approach was found to be important, encouraging patients to get in touch with any problems or questions. Other helpful strategies included highlighting the benefits of insulin-explaining that it is a natural substance the body needs-and addressing any specific concerns a patient may have about insulin. The least helpful strategy was taking an authoritarian approach and repeatedly trying to persuade a patient to start insulin. This is the first study which provides evidence-based clinical strategies that UK healthcare professionals can use to help overcome PIR in their patients with T2DM.

Keywords: Basal insulin; Healthcare professionals; Psychological insulin resistance; Type 2 diabetes mellitus; UK patients

\section{Key Summary Points}

Why carry out this study?

Patients with type 2 diabetes mellitus (T2DM) are often reluctant to initiate insulin recommended by their healthcare professionals (HCPs).

This phenomenon is termed 'psychological insulin resistance'.

\section{What was learned from this study?}

We identify key strategies by HCPs in the UK that most helped their patients.

The most helpful strategy was demonstrating the injection process to the patients.

The least helpful action was HCPs repeatedly persuading patients to initiate insulin.

\section{INTRODUCTION}

Diabetes mellitus, a chronic disease, involves either impaired insulin production or insulin ineffectiveness. According to Diabetes UK [1], approximately 3.8 million people have been diagnosed with diabetes mellitus in the United Kingdom (UK). Of these, almost 90\% have type 2 diabetes mellitus (T2DM) [1]. Disease progression, and specifically poor glycaemic control, eventually results in several debilitating and/or life-threatening complications [2, 3]. Early initiation of insulin in T2DM is important in order to address poor glycaemic control and prevent the development of diabetes-related complications [4].

Despite this, a sizeable body of data highlights a delay in initiation of insulin therapy. In a population-based analysis in the UK, we observed a delay of almost 5 years in insulin initiation in $50 \%$ of adults who had T2DM with glycated haemoglobin $\quad(\mathrm{HbA} 1 \mathrm{c}) \geq 8 \%$ $(63.9 \mathrm{mmol} / \mathrm{mol})$ and diabetes-related complications [5]. More than $30 \%$ of patients with T2DM showed reluctance to initiate insulin therapy when recommended by their healthcare professionals (HCPs) to do so [6-8]. This phenomenon has been referred to as 'psychological insulin resistance' (PIR) [8-10]. Previous studies have identified various factors accounting for PIR, such as lack of knowledge on physiology or guidelines, negative self-perceptions, attitudinal barriers, lack of time or resources, social stigmatization and fear of 
injection and/or side effects, including hypoglycaemia [10, 11]. Identifying and eventually overcoming PIR is critical to diabetes management. Some studies mention ways to reduce PIR [12-15], but few have discussed approaches to overcoming this resistance in detail. The present research seeks to elucidate this understanding by identifying patient-HCP interactions that helped patients with initial insulin reluctance to eventually begin using insulin. Documenting these strategies could help HCPs facilitate an easier transition to insulin use and reduce the time between when HCPs first recommend insulin and when patients initiate it.

The EMOTION (AccEpting Insulin TreatMent for Reluctant PeOple with Type 2 DIabetes Mellitus-A GlObal Study to IdeNtify Effective Strategies) study surveyed adults with T2DM and PIR in the United States (USA), UK, Canada, Germany, Spain, Brazil and Japan. The objective of this study was to evaluate the perceived occurrence and helpfulness of HCPs' statements and actions that assisted these participants to begin basal insulin. The EMOTION study identified three key HCP actions that were associated with more successful insulin therapy initiation and persistence: demonstrating the injection process, explaining the benefits of insulin and adopting a collaborative communication approach [16]. We understand that the frequency and helpfulness of HCP strategies might vary by country. Therefore, we present here the results from a subgroup of the EMOTION study, focusing on UK adults to help UKbased clinicians understand the most and least helpful statements and actions in their own country.

\section{METHODS}

\section{Study Design and Participants}

Adults (aged $\geq 21$ years) diagnosed with T2DM for at least 1 year before initiating basal insulin and presently taking basal insulin for a minimum of 30 days and up to 3 years before the online survey were eligible to participate. At screening, these adults reported that they were initially "not willing" or only "slightly willing" to initiate insulin therapy when first asked by HCPs to do so. Adults with type 1 diabetes mellitus or gestational diabetes, or those who initiated premixed or basal bolus as insulin therapy, were excluded. Detailed information on the methodology, including descriptions about the different phases and how they were conducted, as well as the results for the overall population have been presented elsewhere [16].

This manuscript focuses on participants in the UK, who were recruited from either public diabetes website announcements or Survey Sampling International and their partners' market research panels. The participants recruited through diabetes website announcements were not offered any compensation for completing the survey, whereas participants recruited from market research panels received the compensation offered generally for surveys of similar duration. The work described was carried out in accordance with the Code of Ethics of the World Medical Association (Declaration of Helsinki), the protocol was reviewed by a central Institutional Review Board (IRB)Western IRB (Puyallup, WA, USA) and informed consent was obtained for all participants.

\section{Measures}

The PIR Action Survey (PAS) that was administered as a part of the broader 30-min online survey in the EMOTION study assessed the perceived occurrence and helpfulness of 38 statements and actions by HCPs about insulin initiation that may have influenced a participant's decisions regarding insulin therapy. Each item on the PIR Action Survey was rated on a 5-point Likert scale: 0 (did not occur), 1 (did occur, and didn't help at all), 2 (did occur, and helped a little), 3 (did occur, and helped moderately) and 4 (did occur, and helped a lot). Details about the development of the 38-item questionnaire have been previously reported [16].

The broader 30-min survey also collected participants' demographic data and clinical characteristics, including self-reported most recent HbA1c and prior injectable anti- 
hyperglycaemic medication use (such as a glucagon-like peptide 1 receptor agonist), if any. Time to actual insulin initiation following the initial recommendation and insulin persistence since it was first initiated were also assessed. A single question evaluated the time to initiation: "How much time passed between when your HCP first recommended that you start taking insulin and when you actually started taking it regularly?" Eight response options were offered: 'I started taking it right away', 'less than 1 week', 'about 1 or 2 weeks', 'about 1 month', ' 2 to 3 months', ' 4 to 6 months', ' 7 to 12 months' and 'more than 1 year'. Any response other than 'I started taking it right away' was viewed as a delay in initiation. A single yes/no question measured insulin persistence: "Since first starting insulin, has there been a period of 7 or more days when you did not use any kind of insulin?"

\section{Statistical Analyses}

Findings from the UK participants were summarised using descriptive statistics. As the objective of this study was not intended to be a direct comparison, statistical inferences and comparisons across countries were not made. The frequency and percentage of PIR Action Survey item occurrence were evaluated by dichotomising the response options as 0 (did not occur) and 1 (did occur). The frequency and percentage of item helpfulness was evaluated by dichotomising the 4-point score for those items that occurred: 'not helpful' $(1=$ not helpful or 2 = helped a little) and 'helpful' ( $3=$ helped moderately or $4=$ helped a lot).

\section{RESULTS}

Between December 2016 and August 2017, a total of 594 adults with T2DM meeting the eligibility criteria participated in the EMOTION study [16]. Of these, 125 participants were recruited in the UK: 39 from the market research panels and 86 through public diabetes website announcements. The mean age of UK participants was 53.9 years, and they initiated insulin an average of 9.5 years after T2DM
Table 1 Participants' characteristics

\begin{tabular}{ll}
\hline Characteristic & $N=\mathbf{1 2 5}$ \\
\hline Age & $53.9 \pm 10.9$ \\
Male & $50(40.0)$ \\
Years since first T2DM diagnosis to insulin & $9.5 \pm 7.3$ \\
initiation & \\
BMI prior to insulin initiation, kg/m ${ }^{2}$ & $31.6 \pm 7.9$ \\
BMI category & \\
$<25.0$ & $26(20.8)$ \\
$\geq 25.0$ to $<30.0$ & $31(24.8)$ \\
$\geq 30.0$ & $66(52.8)$ \\
Most recent self-reported HbAlc value & \\
\% & $7.6 \pm 2.2$ \\
mmol/mol & $59.1 \pm 23.6$ \\
HbAlc self-report value prior to insulin initiation \\
\%
\end{tabular}

Data are presented as mean \pm standard deviation or $n(\%)$ unless otherwise indicated

$B M I$ body mass index, HbAlc glycated haemoglobin, $T 2 D M$ type 2 diabetes mellitus

diagnosis. Mean self-reported $\mathrm{HbA1c}$ at the time of the survey was $7.6 \%(59.1 \mathrm{mmol} / \mathrm{mol})$, and $\mathrm{HbA1c}$ at the time of insulin initiation was $9.9 \%$ $(84.9 \mathrm{mmol} / \mathrm{mol}$; Table 1$)$.

When first asked by HCPs to take insulin, most participants were either very $(16.8 \%)$, moderately $(16.0 \%)$ or slightly $(36.0 \%)$ surprised, whereas only $10.4 \%$ of patients were not 
Table 2 Participants' reactions to HCPs' recommendations to initiate insulin $(N=125)$

Reaction to recommendation to initiate $n(\%)$
insulin

Level of surprise when HCP first said it was time to take insulin

Not surprised at all

$39(31.2)$

Slightly surprised

$45(36.0)$

Moderately surprised

$20(16.0)$

Very surprised

$21(16.8)$

Level of being upset when HCP first said it was time to take insulin

Not upset at all

$13(10.4)$

Slightly upset

$50(40.0)$

Moderately upset

Very upset

$33(26.4)$

Willingness to start insulin when first recommended

Not willing

Slightly willing

Reaction to HCP first saying it was time to start insulin

Patient started insulin right away

$71(56.8)$

Patient took the prescription, but didn't get it 12 (9.6) filled immediately

Patient got the insulin, but didn't start taking 13 (10.4) it immediately

Patient asked for more time

Patient told HCP that he/she just couldn't 6 (4.8) do it

Time between HCP recommendation and insulin initiation

Started taking it right away

Less than 1 week

1-2 weeks

$20(16.0)$

1 month

2-3 months

$4(3.2)$

4-6 months

$2(1.6)$

$>6$ months

$6(4.8)$
Table 2 continued

Reaction to recommendation to initiate $n(\%)$
insulin

One or more periods of discontinuing insulin $5(4.0)$

for $\geq 7$ days

$H C P$ healthcare professional

upset at all. Further, $51.2 \%$ of participants were slightly willing to initiate insulin and the rest were not willing to start insulin at all (Table 2). Although each participant was reluctant to initiate insulin to some extent, $56.8 \%$ of participants started taking insulin immediately, and $10.4 \%$ initiated insulin in less than 1 week after recommendation by HCP. Following insulin initiation, $4 \%$ of the participants indicated one or more periods of discontinuing insulin for at least 7 days.

The most commonly occurring actions in more than $90 \%$ of participants were the HCP showing the patient an insulin pen, the HCP talking the patient through the process on how to take insulin, the HCP discussing with the patient that blood glucose numbers would improve with insulin and the HCP encouraging the patient to contact his/her clinic immediately in case of any problems/questions. Figure 1 summarises the eight most and eight least helpful strategies by HCPs that facilitated participants to initiate insulin as reported in the survey; these were found to help at least $77 \%$ of participants moderately or a lot and were considered slightly or not at all helpful by at least $36 \%$ of participants. The most helpful HCP statement/action was "HCP talked the patient through the whole process of exactly how to take insulin" (83.6\% of participants considered this action moderately or very helpful; occurrence was reported by $92.8 \%$ of participants [Fig. 1a, Table S1 in the electronic supplementary material]). The least helpful HCP statement/action was "Repeatedly over many visits, HCP kept trying to convince the patient to get started on insulin" $(59.1 \%$ of participants 
(a)

HCP talked patient through the whole process of exactly how to take insulin

HCP explained that insulin was a natural substance that the patient's body needed

HCP helped patient to see how simple it was to inject insulin

HCP encouraged the patient to contact his/her clinic immediately if the patient ran into any problems or had questions after starting insulin

HCP reviewed patient's blood sugar numbers with the patient, showing the patient that his/her diabetes was not under control and that action was needed

HCP explained that the patient might not have to take insulin forever

HCP had patient try an injection himself/herself while patient was there in the clinic

HCP showed patient an insulin pen

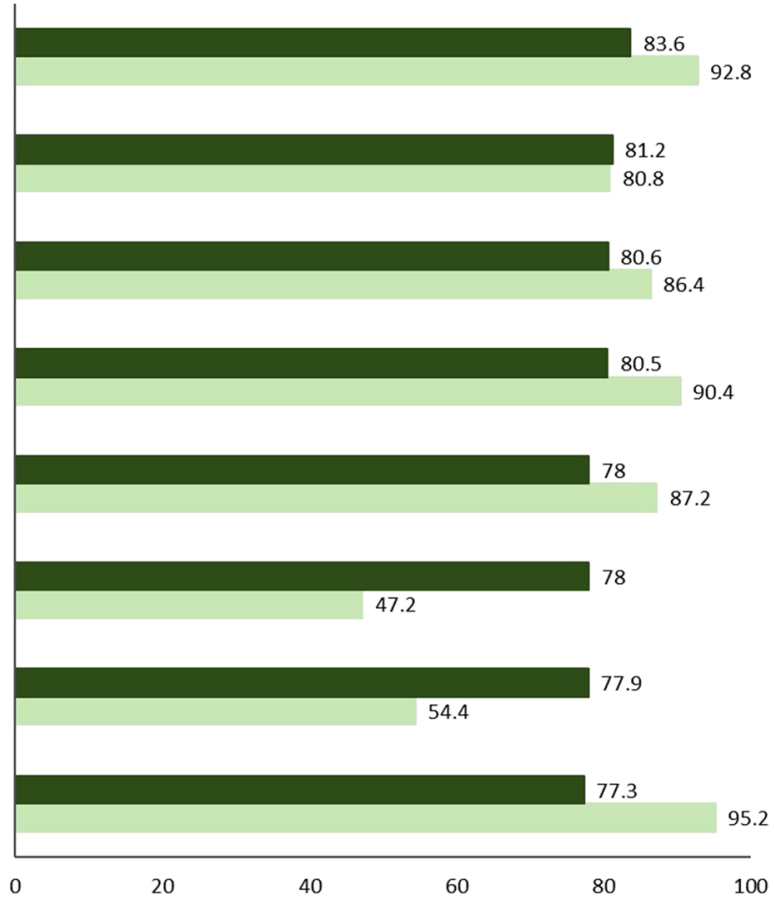

\% of patients with occurrence (b)

Repeatedly over many visits, HCP kept trying to convince the patient to get started on insulin

HCP referred patient to a class to help learn more about insulin

$\mathrm{HCP}$ reassured the patient that he/she would help the patient avoid or minimise any weight gain because of taking insulin

HCP told patient that he/she just needed to trust that the HCP knew best and that getting started on insulin was the patient's best option

HCP helped patient meet other people who had already been taking insulin for a while

HCP helped patient to realise that insulin wasn't going to cost patient as much money as the patient feared it would

HCP helped patient to see that an insulin injection wasn't as painful as patient thought it might be

HCP told patient about all of the positives and negatives of insulin, and explained how the positives outweighed the negatives

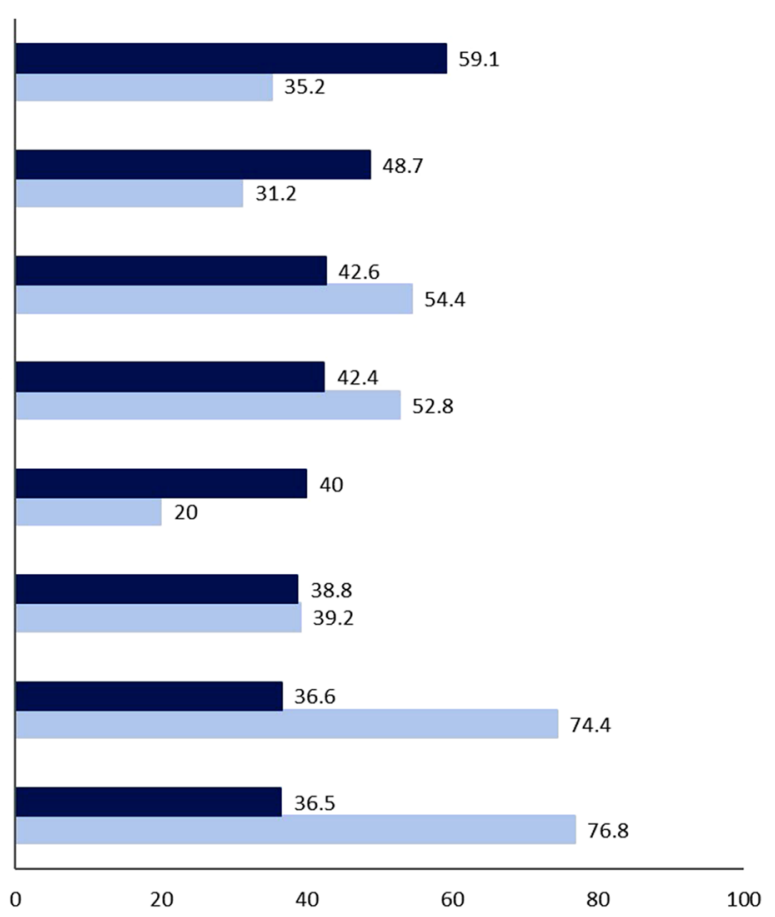

\% of patients with occurrence 
4Fig. 1 Eight a most and $\mathbf{b}$ least helpful strategies in insulin initiation among participants with T2DM reporting an item as having occurred. Note: Each item on the PIR Action Survey was rated as 0 (did not occur), 1 (did occur, and didn't help at all), 2 (did occur, and helped a little), 3 (did occur, and helped moderately) or 4 (did occur, and helped a lot); the percentage of item helpfulness was evaluated among participants for whom the action occurred. HCP healthcare, PIR psychological insulin resistance, $T 2 D M$ type 2 diabetes mellitus

considered this action slightly or not at all helpful; occurrence was reported by $35.2 \%$ of participants [Fig. 1b, Table S1 in the electronic supplementary material]). Table $\mathrm{S} 1$ in the electronic supplementary material provides additional details on HCPs' statements and actions that were analysed in the PIR Action Survey, along with their occurrence and helpfulness.

Interestingly, $60 \%$ of participants were either satisfied or very satisfied with their current insulin treatment by the time the survey was conducted. Additionally, $28.8 \%$ of participants had neutral feelings towards the insulin treatment, whereas only $11.2 \%$ were either unsatisfied or not at all satisfied with ongoing insulin treatment.

\section{DISCUSSION}

The present research explored the frequency and helpfulness of HCPs' statements and actions that helped adults with T2DM in the UK who were initially reluctant to start insulin therapy eventually make a favourable decision. It was observed in the global survey that the clinicians utilised a variety of strategies to help patients overcome the insulin initiation resistance. However, participants perceived not all actions as equally helpful [16].

In the UK subgroup, the majority of the most helpful statements and/or actions were related to the factor "demonstration of the injection process" identified in the overall study and considered to be directed towards reducing fears and discomfort about the injection procedure. Of interest, this factor also presented the highest mean levels of helpfulness in the overall study [16]. These statements and actions included the following: HCP talking the patient through the whole process on exactly how to take insulin, HCP showing the patient an insulin pen, HCP helping the patient try an injection himself/herself during their consultation and HCP helping the patient see how simple it was to inject insulin. These actions were reported as occurring more frequently. Additionally, these strategies were consistent with clinical recommendations commonly utilised for addressing PIR, for example, the importance of educating patients on injection technique at the time of insulin initiation (such as simple and straightforward instructions on when and how to take an injection, including site rotation and disposal) [17]. Further, these data correlate with the findings from the TRIAD (Translating Research Into Action for Diabetes Insulin Starts) project, where patients who did not begin insulin therapy commonly reported limited/a lack of self-management training [18].

Other strategies that extensively helped participants were HCPs explaining that insulin was a natural substance that the body needed, encouraging patients to contact them immediately in case of any problems/questions, presenting to patients their blood glucose numbers and helping them understand that their diabetes was not under control and needing action. A recent study in Canada in patients with T2DM not achieving recommended glycaemic targets provided similar practical recommendations to HCPs, highlighting that explaining the benefits of insulin therapy, allaying patients' concerns and collaborating with patients to explain the need for insulin were the key strategies to help patients overcome PIR [19]. Other studies that underlined the importance of $\mathrm{HCP}$-patient collaboration to develop a shared action plan reported not only positive patient insulin initiation experiences but also improved concordance with insulin therapy $[17,20]$.

Consistent with the findings in the overall study report, HCPs' statements and actions linked to an authoritarian communication approach that was identified in the overall study, such as HCPs repeatedly trying to convince patients to start insulin, were observed to be least helpful in the UK [16]. These actions were reported to occur less frequently and were 
broadly considered to result in poorer outcomes.

To date, various studies in the UK have evaluated the evidence and reasons for clinical inertia and underlined the importance of selfmanagement educational programmes and clinician education and training $[21,22]$. However this is the first study specific to adults with T2DM in the UK which provides evidence-based clinical recommendations/strategies to HCPs for addressing PIR in such patients.

The study does have some limitations. Owing to the nature of the study, participants were recruited from online panels and through public diabetes website announcements; we could assume these adults were possibly more involved with their condition than the usual patient population in the country would be. Thus, the group of adults who showed willingness to participate in the study were possibly not the most reluctant group of patients, and the findings may not be completely true to the entire community with T2DM exhibiting PIR. Bias may also have been introduced as a result of the differing levels of compensation offered to participants dependent on their mode of recruitment. However, as $69 \%$ of UK participants in the study were recruited through the same non-payment method, any bias due to receipt of incentives is likely to be limited. Further, these participants were younger than expected so might not be representative of all adults with T2DM in the UK. The data for events that occurred previously were selfreported and might be subject to recall bias. The verification of variables such as the presence of T2DM and the time of insulin initiation using chart records was not carried out. Moreover, the descriptive design of the study may not adjust for plausible confounders. Finally, the small sample size could have biased the results, undermining the external validity of the study, and results should be interpreted with caution. Replicating the study in a sufficiently large and diverse sample is warranted.

\section{CONCLUSIONS}

This study provides evidence-based clinical recommendations/strategies that HCPs can use to help patients with T2DM overcome PIR. Given the age range of the UK EMOTION subgroup, these results may prove more applicable in a younger patient population than would typically be expected in T2DM. HCPs' statements and actions perceived by participants to be most helpful to address PIR were related to demonstrating the injection procedure, adopting a collaborative approach, highlighting the benefits of insulin and allaying/dispelling patients' concerns/myths. Utilising an authoritarian communication approach was reported to be the least helpful action.

\section{ACKNOWLEDGEMENTS}

We thank the participants of the study.

Funding. This study was sponsored by Eli Lilly and Company. The study sponsor is also funding the journal's Rapid Service Fee.

Medical Writing and Editorial Assistance. Medical writing assistance in the preparation of this article was provided by Richi Taneja of Eli Lilly and Company (Bengaluru, India). Editorial assistance was provided by $\mathrm{Rx}$ Communications (Mold, UK) and funded by Eli Lilly and Company.

Authorship. All named authors meet the International Committee of Medical Journal Editors (ICMJE) criteria for authorship for this article, take responsibility for the integrity of the work as a whole, and have given their approval for this version to be published.

Disclosures. Magaly Perez-Nieves, Dachuang Cao and Irene Hadjiyianni are employees and stockholders of Eli Lilly and Company. Elemer Balogh (Abbott Laboratories Limited, UK) and Nadeem Ashraf (Boehringer Ingelheim, UK) are former employees of Eli Lilly and Company. Urvi Desai is an employee of Analysis Group, 
Inc., a company that received funding for this research from Eli Lilly and Company. Frank J Snoek was consultant and speaker for Eli Lilly and Company during the conduct of this study, has consulted for Novo Nordisk and Roche Diabetes Care and received research grants from Sanofi. Fees and grants were all paid to the institute [Amsterdam UMC]. Jackie A. Sturt received consultancy and/or speaker fees from Eli Lilly and Spirit Healthcare.

Compliance with Ethics Guidelines. The work described was carried out in accordance with the Code of Ethics of the World Medical Association (Declaration of Helsinki), the protocol was reviewed by a central Institutional Review Board (IRB)-Western IRB (Puyallup, WA, USA) and informed consent was obtained for all participants.

Data Availability. No data are available as the information collected during patient surveys is considered confidential, and disclosure to third parties is not permitted.

Open Access. This article is licensed under a Creative Commons Attribution-NonCommercial 4.0 International License, which permits any non-commercial use, sharing, adaptation, distribution and reproduction in any medium or format, as long as you give appropriate credit to the original author(s) and the source, provide a link to the Creative Commons licence, and indicate if changes were made. The images or other third party material in this article are included in the article's Creative Commons licence, unless indicated otherwise in a credit line to the material. If material is not included in the article's Creative Commons licence and your intended use is not permitted by statutory regulation or exceeds the permitted use, you will need to obtain permission directly from the copyright holder. To view a copy of this licence, visit http://creativecommons.org/licenses/by$\mathrm{nc} / 4.0 /$.

\section{REFERENCES}

1. Diabetes UK. Us, diabetes and a lot of facts and stats. London: Diabetes UK. Available from https:// www.diabetes.org.uk/professionals/positionstatements-reports/statistics. Accessed 12 June 2020

2. Nickerson HD, Dutta S. Diabetic complications: current challenges and opportunities. J Cardiovasc Transl Res. 2012;5:375-9.

3. Pantalone KM, Misra-Hebert AD, Hobbs TM, et al. Effect of glycemic control on the Diabetes Complications Severity Index score and development of complications in people with newly diagnosed type 2 diabetes. J Diabetes. 2018;10:192-9.

4. Mashitisho MLI, Mashitisho B. Early insulin therapy in patients with type 2 diabetes mellitus. JEMDSA. 2016;21:13-5.

5. Rubino A, McQuay LJ, Gough SC, Kvasz M, Tennis P. Delayed initiation of subcutaneous insulin therapy after failure of oral glucose-lowering agents in patients with type 2 diabetes: a population-based analysis in the UK. Diabet Med. 2007;24:1412-8.

6. Hosomura N, Malmasi S, Timerman D, et al. Decline of insulin therapy and delays in insulin initiation in people with uncontrolled diabetes mellitus. Diabet Med. 2017;34:1599-602.

7. Polonsky WH, Fisher L, Guzman S, Villa-Caballero L, Edelman SV. Psychological insulin resistance in patients with type 2 diabetes: the scope of the problem. Diabetes Care. 2005;28:2543-5.

8. Polonsky WH, Hajos TR, Dain MP, Snoek FJ. Are patients with type 2 diabetes reluctant to start insulin therapy? An examination of the scope and underpinnings of psychological insulin resistance in a large, international population. Curr Med Res Opin. 2011;27:1169-74.

9. Abu Hassan H, Tohid H, Mohd Amin R, Long Bidin MB, Muthupalaniappen L, Omar K. Factors influencing insulin acceptance among type 2 diabetes mellitus patients in a primary care clinic: a qualitative exploration. BMC Fam Pract. 2013;14:164.

10. Brod M, Kongso JH, Lessard S, Christensen TL. Psychological insulin resistance: patient beliefs and implications for diabetes management. Qual Life Res. 2009;18:23-32.

11. Gherman A, Veresiu IA, Sassu RA, Schnur JB, Scheckner BL, Montgomery GH. Psychological insulin resistance: a critical review of the literature. Pract Diabetes Int. 2011;28:125-8d. 
12. Nam S, Chesla C, Stotts NA, Kroon L, Janson SL. Factors associated with psychological insulin resistance in individuals with type 2 diabetes. Diabetes Care. 2010;33:1747-9.

13. Racz O, Linkova M, Jakubowski K, Link R, Kuzmova D. Barriers of the initiation of insulin treatment in type 2 diabetic patients-conquering the "psychological insulin resistance". Orv Hetil. 2019;160: 93-7.

14. Aleali AM, Payami SP, Latifi SM, Yazdanpanah L, Hesam S, Khajeddin N. Evaluation of psychological resistance to insulin treatment in type II diabetic patients. Diabetes Metab Syndr. 2018;12:929-32.

15. Allen NA, Zagarins SE, Feinberg RG, Welch G. Treating psychological insulin resistance in type 2 diabetes. J Clin Transl Endocrinol. 2017;7:1-6.

16. Polonsky WH, Fisher L, Hessler D, et al. Identifying solutions to psychological insulin resistance: an international study. J Diabetes Complic. 2019;33: 307-14.

17. Polonsky WH, Arsenault J, Fisher L, et al. Initiating insulin: how to help people with type 2 diabetes start and continue insulin successfully. Int J Clin Pract. 2017;71:8.

18. Karter AJ, Subramanian U, Saha C, et al. Barriers to insulin initiation: the translating research into action for diabetes insulin starts project. Diabetes Care. 2010;3:733-5.

19. Berard L, Antonishyn N, Arcudi K, et al. Insulin matters: a practical approach to basal insulin management in type 2 diabetes. Diabetes Ther. 2018;9: 501-19.

20. Linetzky B, Jiang D, Funnell MM, Curtis BH, Polonsky WH. Exploring the role of the patientphysician relationship on insulin adherence and clinical outcomes in type 2 diabetes: insights from the MOSAIc study. J Diabetes. 2017;9:596-605.

21. Russell-Jones D, Pouwer F, Khunti K. Identification of barriers to insulin therapy and approaches to overcoming them. Diabetes Obes Metab. 2018;20: 488-96.

22. Khunti K, Millar-Jones D. Clinical inertia to insulin initiation and intensification in the UK: a focused literature review. Prim Care Diabetes. 2017;11:3-12. 